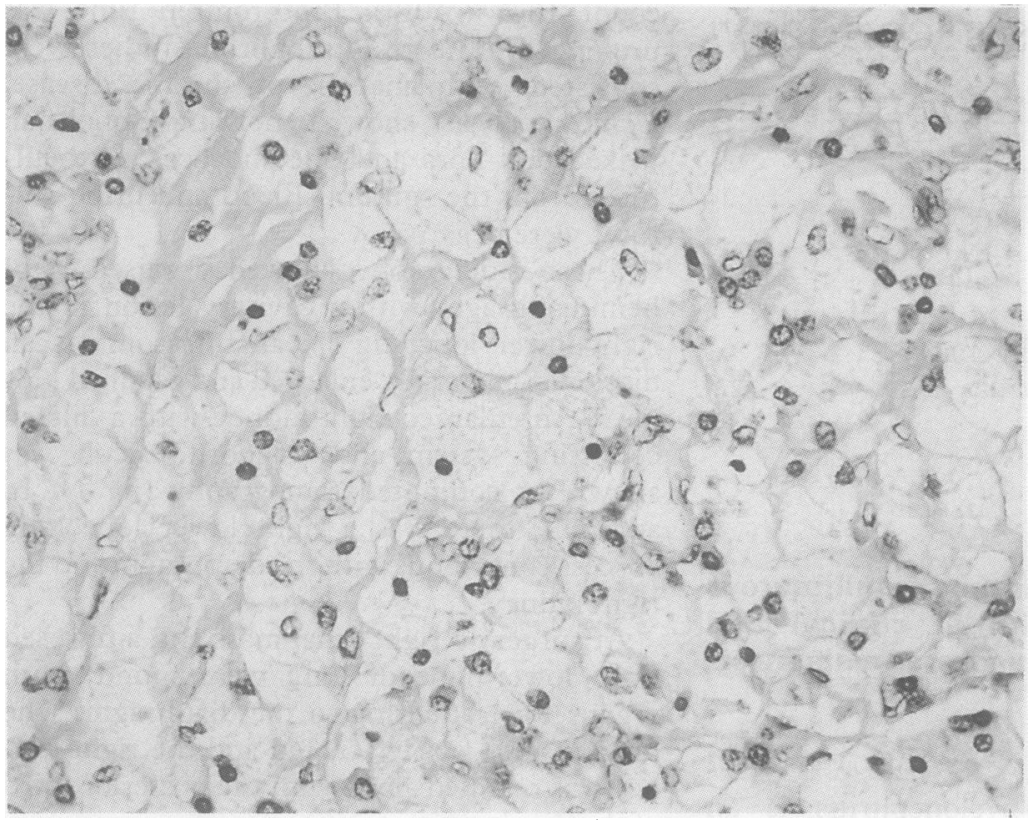

Figure 2 Microscopic section of the lesion showing the sheet like arrangement of numerous, rounded histiocytes with the abundant, foamy, clear cytoplasm and scattered plasma cells characteristic of xanthogranulomatous pyelonephritis.

phragm and has been reported, albeit rarely, to form fistulous tracts with microscopic lesions of the pleura, no case has been described where a lung abscess caused the patient's initial presentation.' Because our patient had no abnormal abdominal findings, we originally diagnosed a lung abscess on the basis of the chest radiograph. Xanthogranulomatous pyelonephritis was discovered during investigation of the raised alkaline phosphatase activity.

The aetiology of xanthogranulomatous pyelonephritis is not known but it has been produced experimentally in rats by ureteral ligation and intravenous administration of a specific Escherichia coli serotype. ${ }^{2}$ In man the most frequently encountered organisms are $E$ coli and Proteus mirabilis. ${ }^{2}$ Surgical removal of the affected kidney is the treatment of choice in most cases.

1 Parsons MA, Harris SC, Grainger RG, Ross B, Smith JAR, Williams $J L$. Fistula and sinus formation in xanthogranulomatous pyelonephritis. $B r J$ Urol 1986;58:488-93.

2 Cohen MS. Granulomatous nephritis. Urol Clin North Am 1986;13:647-59.

3 Malek RS, Elder JS. Xanthogranulomatous pyelonephritis: a critical analysis of 26 cases and of the literature. $J$ Urol a critical analysis

4 Grainger RG, Longstaff AJ, Parsons MA. Xanthogranulomatous pyelonephritis: a reappraisal. Lancet 1982;i:1398-401.
Service of Clinical Pathology L Fogue

Hospital Ramón y Cajal, Universidad de Alcalá de Henares, Madrid, Spain F Ruiz-Palomo J L Calleja

Address for reprint requests: Dr F Ruiz-Palomo, Dr F Ruiz-Paic

Homero 44, 2820 , Madrid, Spain.

Accepted 11 September 1989

\section{Primary liposarcoma of the lung in a young} woman

\author{
F Ruiz-Palomo, J L Calleja, L Fogue
}

\begin{abstract}
A primary liposarcoma of the lung occurred in an 18 year old girl, the first to be reported in anyone under 40 . Though rare, intrathoracic liposarcoma should be included in the differential diagnosis of pleural effusion in younger patients.
\end{abstract}

Liposarcoma is one of the more common soft tissue neoplasms of adult life, ${ }^{1}$ but it is rare in patients under 40 years of age, and has rarely been reported in anyone under $20 .^{2}$ The most frequent primary sites are the extremities and the retroperitoneum. An intrathoracic origin is unusual, and most arise in the mediastinum, ${ }^{23}$ though pleural and pulmonary tumours have been described. ${ }^{45}$ The chest was affected in only 29 of 1067 cases from the files of the United States Armed Forces Institute of Pathology. ${ }^{2}$ We describe a case of primary liposarcoma of the lung in a young woman of 18 years.

\section{Case report}

An 18 year old woman was admitted for investigation of a massive left pleural effusion. She had had a non-productive cough for three weeks and progressive dyspnoea on exertion for five days. Six days before admission she had had an episode of pyrexia $\left(38^{\circ} \mathrm{C}\right)$, which had resolved spontaneously. She denied weight loss; anorexia, drug addiction, contact with pets, allergies, articular pain, and previous tuberculosis. She had smoked 10 cigarettes a day for three years, and had had brucellosis four years previously.

On examination she appeared well, but had signs of a left sided pleural effusion. The haemoglobin concentration was $12.9 \mathrm{~g} / \mathrm{dl}$, the total white cell count $12.5 \times 10^{9} / 1$, and the erythrocyte sedimentation rate $65 \mathrm{~mm}$ in one hour. There was no biochemical abnormality. A chest radiograph showed a large left pleural effusion displacing the cardiac silhouette to the right. No malignant cells or tubercle bacilli were found on examination of the sputum. Pleural aspiration yielded a slightly blood stained fluid with a $\mathrm{pH}$ of $7 \cdot 31$, a glucose concentration of $4.2 \mathrm{mmol} / 1$, a total protein concentration of $44 \mathrm{~g} / 1$ and a white cell count of $3.14 \times 10^{9} / 1$, with a predominance of lymphocytes and mononuclear cells. No organisms were shown by Gram staining, and mycobacteria were not found. A pleural biopsy specimen showed non-specific inflammatory changes, with no evidence of malignancy. Abdominal ultrasonography showed no abnormality and bronchoscopy showed only extrinsic compression of the left bronchial tree. 
Treatment with antituberculous drugs was started, but the patient's condition continued to deteriorate and she developed a pyrexia of $38.5^{\circ} \mathrm{C}$ and dyspnoea at rest. Intercostal drainage yielded a blood stained, jelly like material, which frequently blocked the tube. Despite these measures there was no improvement, and thoracotomy was carried out. At operation a large, partially necrotic tumour was found in the left upper lobe, extending into the left lower lobe, parietal pleura, and mediastinum. Its cut surface was yellow and its consistency friable. Unfortunately, during the operation the patient suffered a cardiac arrest and attempts at resuscitation were unsuccessful.

Microscopically the tumour consisted of proliferating lipoblasts, with considerable nuclear pleomorphism, in a stroma containing a rich capillary network (figure). A study with immunoperoxidase tumour markers was made to exclude a non-lipomatous origin: reactions for vimentin, desmin, myoglobin, $\alpha_{1}$ antitrypsin, cytokeratins, carcinoembryonic antigen, $\alpha$ fetoprotein, and common leucocyte antigen all gave negative results. The diagnosis of liposarcoma was confirmed by electron microscopy.

\section{Discussion}

Liposarcoma is rare in childhood and adolescence. In a recent review of the published reports Shmookler and Enzinger found only 29 cases in patients under 16 years of age, ${ }^{2}$ only one of which was located in the chest. ${ }^{6}$ Only seven cases of pulmonary liposarcoma had been reported by $1982 .^{5}$

In our case a pulmonary origin appears to be the most likely. There was no evidence that the tumour had originated in the chest wall, and the mediastinal tumour seemed to be a consequence of spread from the lung. An abdominal origin could also be ruled out because the abdominal ultrasound scan was normal, and as there was no palpable tumour in the extremities metastatic spread could be excluded.

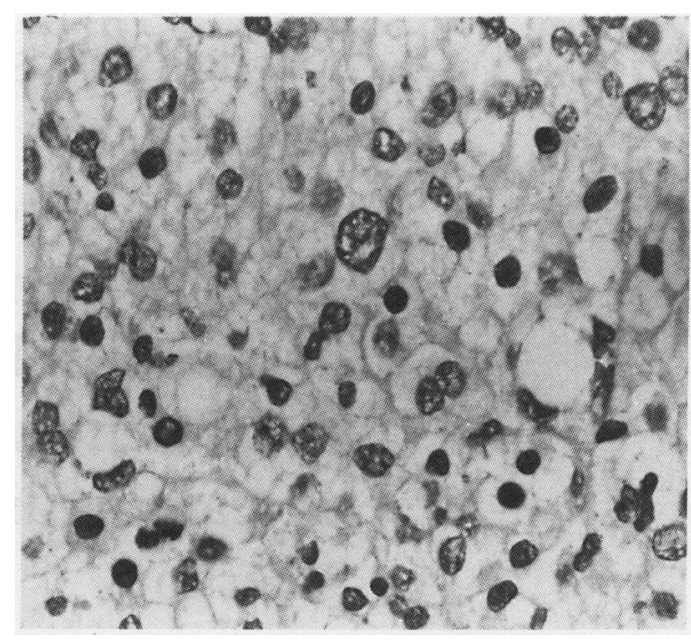

Histological appearance of tumour, showing proliferation of lipoblasts with presence of vacuolation and nuclear pleomorphism. (Haematoxylin and eosin.)

So far as we are aware, this is the first primary pulmonary liposarcoma to be reported in a patient under 40 years of age. During the first few days after admission pleuropulmonary tuberculosis was thought to be the most likely diagnosis, given the high prevalence of this condition in Spain. It was only the patient's rapid deterioration despite antituberculous treatment that indicated the neoplastic nature of the disease. Intrathoracic liposarcoma, although extremely rare, should be included in the differential diagnosis of pleural effusion in this age group.

1 Enzinger FM, Sharon WW. Soft tissue tumours. St Louis: Mosby, 1983:242-50.

2 Shmookler BM, Enzinger FM. Liposarcoma occurring in children: an analysis of 17 cases and review of the literature. Cancer 1983;52:567-74.

3 Razzak MA, Urshel HC, Race GJ, et al. Liposarcoma of the mediastinum. Case report and review of the literature. $J$ Thorac Cardiovasc Surg 1971;61:819-26.

4 Evans AR, Wolstenholme RJ, Shettar SP, Yogish $\mathrm{H}$. Primary pleural liposarcoma. Thorax 1985;40:554-5.

5 Sawamura K, Hashimoto $T$, Nanjo S, et al. Primary liposarcoma of the lung: report of a case. J Surg Oncol 1982;19:243-6.

6 Kauffman SC, Stout AP. Lipoblastic tumours of children. Cancer 1959;12:912-25. 